\title{
Crossover from Majorana edge- to end-states in quasi-one-dimensional $p$-wave superconductors
}

\author{
Bin Zhou ${ }^{1,2}$ and Shun-Qing Shen ${ }^{2}$ \\ ${ }^{1}$ Department of Physics, Hubei University, Wuhan 430062, China \\ ${ }^{2}$ Department of Physics and Center for Theoretical and Computational Physics, The University of Hong Kong, \\ Pokfulam Road, Hong Kong, China
}

(Received 9 May 2011; revised manuscript received 12 July 2011; published 11 August 2011)

\begin{abstract}
In a recent work [Potter and Lee, Phys. Rev. Lett. 105, 227003 (2010)], it was demonstrated by means of numerical diagonalization that the Majorana end states can be localized at opposite ends of a sample of an ideal spinless $p$-wave superconductor with the strip geometry beyond the strict one-dimensional limit. Here, we reexamine this issue and study the topological quantum phase transition in the same system. We give the phase diagrams of the presence of Majorana end modes by using of $Z_{2}$ topological index. It is found that the topological property of a strip geometry will change in an oscillatory way with respect of the sample width.
\end{abstract}

DOI: 10.1103/PhysRevB.84.054532

PACS number(s): 71.10.Pm, 74.20.Rp, 74.78.-w

\section{INTRODUCTION}

Recently, there is a growing interest in searching for Majorana fermions in the condensed matter physics community. ${ }^{1}$ Unlike traditional fermions, Majorana fermions are their own antiparticles and are expected to obey non-Abelian statistics. ${ }^{2,3}$ Due to novel properties of the Majorana particles, it has been proposed that they can be used in realizing the faulttolerant topological quantum computation protected from local decoherence. ${ }^{4-8}$ The early experimental efforts were focused on the $\gamma=5 / 2$ fractional quantum Hall state, ${ }^{9,10}$ in which the Majorana fermions are expected to be charge $e / 4$ quasiholes. Other proposed candidate materials include the $p$-wave superconductors, ${ }^{2,11-13}$ in which the Majorana fermions are zero-energy single-particle states trapped in vortex cores and superfluids in the ${ }^{3} \mathrm{He}-B$ phase. ${ }^{14,15} \mathrm{Re}-$ cently, several new suggestions have been proposed to look for Majorana fermions, such as topological insulators proximate to an $s$-wave superconductor, ${ }^{16-24}$ a semiconducting thin film sandwiched between an $s$-wave superconductor and a magnetic insulator, ${ }^{25-27}$ one-dimensional (1D) semiconductor-superconductor heterostructures based on quantum wires, ${ }^{28-30}$ noncentrosymmetric superconductors with broken time-reversal symmetry, ${ }^{31,32}$ and $s$-wave superfluid of ultracold atoms. ${ }^{33}$ Although extensive efforts have been made, direct evidence of Majorana fermions is still absent. Thus, it is still a challenging mission to produce and detect the mysterious Majorana particles.

In his pioneering work, Kitaev ${ }^{5}$ found that Majorana particles can be localized at the ends of a $1 \mathrm{D} p_{x}+i p_{y}$ superconducting wire. In a recent paper, ${ }^{34}$ Potter and Lee moved beyond the strict 1D limit and explored further the notion of Majorana end states in quasi-1D $p_{x}+i p_{y}$ superconductors. They considered a spinless $p$-wave superconductor with finite width, and showed by means of numerical diagonalization that the Majorana end states can be localized at opposite ends of a sample with the strip geometry. We also note that a similar set of ideas on Majorana fermions in the quasi-1D wires was discussed by Wimmer et al. ${ }^{35}$ (see Appendix C of the supplement of Ref. 35). Very recently, searching for Majorana fermions in multiband semiconducting nanowires has attracted much attention and also opens a new path in the challenging field. ${ }^{36-39}$ In this paper, motivated by the work by Potter and Lee, ${ }^{34}$ we reexamine an ideal spinless quasi-1D $p_{x}+i p_{y}$ superconductor and study the topological quantum phase transition in the this system. Firstly, based on the tight-banding model, we give Majorana edge modes localized at boundaries of a sample with the sufficiently large width, and through analytical solutions in a model calculation for a strip of finite width, we find that two Majorana fermions on the two edges can couple together to produce a gap in the excitation spectrum under the periodic boundary condition along the longitudinal direction. Then, we give the phase diagrams of the presence of Majorana end modes in quasi-1D sample by using of $Z_{2}$ topological index. We find that the topological property of a strip geometry will change in an oscillatory way with respect of the sample width.

The paper is organized as follows: in Sec. II, we shall give a square-lattice tight-binding model to describe a spinless $p$-wave superconductor. In Sec. III, we shall investigate the behaviors of Majorana edge states localized at boundaries of the sample with finite width, and the energy gap opened due to the coupling of two Majorana fermions at opposite boundaries is given through analytical solutions in a model calculation. ${ }^{40}$ In Sec. IV, we shall calculate the $Z_{2}$ topological index and plot the phase diagrams of the presence of Majorana end modes in the quasi-1D $p$-wave superconductors. By analyzing the phase diagrams, we shall then give the findings in the topological quantum phase transition in quasi-1D spinless $p$-wave superconductors based on the tight-binding model. We shall summarize our conclusions in Sec. V.

\section{MODEL}

We consider a two-dimensional (2D) Kitaev model of spinless $p$-wave superconductors on a square-lattice, which is described by the following Hamiltonian ${ }^{5,34}$

$$
\begin{aligned}
H= & \sum_{j=1}^{L} \sum_{\alpha=1}^{n}\left[-\mu c_{j, \alpha}^{\dagger} c_{j, \alpha}-\left(t c_{j, \alpha}^{\dagger} c_{j, \alpha+1}+|\Delta| c_{j, \alpha} c_{j, \alpha+1}\right.\right. \\
& \left.\left.+t c_{j, \alpha}^{\dagger} c_{j+1, \alpha}+i|\Delta| c_{j, \alpha} c_{j+1, \alpha}+\text { h.c. }\right)\right],
\end{aligned}
$$

where $c_{j, \alpha}^{\dagger}$ creates an electron on site $(j, \alpha), t(>0)$ is the hopping amplitude, $\mu$ is the chemical potential, $|\Delta|(>0)$ is the $p$-wave pairing amplitude, and the lattice spacing is taken 
as unity. Here, we assume a strip geometry in which the lattice site numbers are $L$ along the $x$-axis direction and $n$ along the $y$-axis direction (the sample width direction), which totals $N=$ $n L$ fermionic sites. First, one introduces a periodic boundary condition along the $x$-axis direction, i.e., $c_{L+1, \alpha}^{\dagger}=c_{1, \alpha}^{\dagger}$, and uses the Fourier transform of the operator $c_{j, \alpha}^{\dagger}$,

$$
c_{j, \alpha}^{\dagger}=\frac{1}{\sqrt{L}} \sum_{q} c_{\alpha}^{\dagger}(q) e^{-i q j},
$$

where $q=q_{x}$ is the wave vector along the $x$-axis, and $-\pi \leqslant$ $q \leqslant \pi$. In terms of the new creation and annihilation operators $c_{\alpha}^{\dagger}(q)$ and $c_{\alpha}(q)$, the Hamiltonian Eq. (1) can be rewritten as

$$
\begin{aligned}
H= & \sum_{q} \sum_{\alpha=1}^{n}\left\{-(\mu+2 t \cos q) c_{\alpha}^{\dagger}(q) c_{\alpha}(q)\right. \\
& -\left[t c_{\alpha}^{\dagger}(q) c_{\alpha+1}(q)+|\Delta| c_{\alpha}(q) c_{\alpha+1}(-q)\right. \\
& \left.\left.+i|\Delta| e^{-i q} c_{\alpha}(q) c_{\alpha}(-q)+\text { h.c. }\right]\right\} .
\end{aligned}
$$

Then, we define a set of the operators $\gamma_{2 \alpha-1}(q)$ and $\gamma_{2 \alpha}(q)$ as

$$
\begin{gathered}
\gamma_{2 \alpha-1}(q)=i\left[c_{\alpha}^{\dagger}(-q)-c_{\alpha}(q)\right], \\
\gamma_{2 \alpha}(q)=c_{\alpha}^{\dagger}(-q)+c_{\alpha}(q),
\end{gathered}
$$

which satisfies the anticommutation relation $\left\{\gamma_{m}^{\dagger}(q), \gamma_{n}\left(q^{\prime}\right)\right\}=$ $2 \delta_{m n} \delta_{q q^{\prime}}$ and $\gamma_{m}^{\dagger}(q)=\gamma_{m}(-q)$. In fact, $\gamma_{m}(0)$ is just a Majorana fermion operator due to $\gamma_{m}^{\dagger}(0)=\gamma_{m}(0)$. In the basis of the news operators $\gamma_{2 \alpha-1}(q)$ and $\gamma_{2 \alpha}(q)$, the Hamiltonian Eq. (3) has the following form:

$$
H=i \frac{1}{4} \sum_{q} \sum_{\eta, \kappa} B_{\eta, \kappa}(q) \gamma_{\eta}(-q) \gamma_{\kappa}(q),
$$

where the elements of the $2 n \times 2 n$ matrix $B(q)$ are given as

$$
\begin{gathered}
B_{2 \alpha, 2 \alpha}=-B_{2 \alpha-1,2 \alpha-1}=-2 i|\Delta| \sin q, \\
B_{2 \alpha, 2 \alpha-1}=-B_{2 \alpha-1,2 \alpha}=-\mu-2 t \cos q, \\
B_{2 \alpha, 2 \alpha+1}=-B_{2 \alpha+1,2 \alpha}=-t-|\Delta|, \\
B_{2 \alpha-1,2 \alpha+2}=-B_{2 \alpha+2,2 \alpha-1}=t-|\Delta|,
\end{gathered}
$$

and the else elements are zero.

If we consider also the periodic boundary condition along the $y$-axis direction, the bulk excitation spectrum of a $2 \mathrm{D}$ square-lattice Kitaev model is given by

$$
\begin{aligned}
E(q)= & \left\{\left[2 t\left(\cos q_{x}+\cos q_{y}\right)+\mu\right]^{2}\right. \\
& \left.+4|\Delta|^{2}\left(\sin ^{2} q_{x}+\sin ^{2} q_{y}\right)\right\}^{1 / 2} .
\end{aligned}
$$

In general, the spectrum Eq. (11) has a gap, but the gap will close when $\mu=-4 t$ at $\mathbf{q}=\left(q_{x}, q_{y}\right)=(0,0)$ or $\mu=4 t$ at $\mathbf{q}=( \pm \pi, \pm \pi)$ or $\mu=0$ at $\mathbf{q}=(0, \pm \pi)$ and $\mathbf{q}=( \pm \pi, 0)$. Actually, $|\mu|=4 t$ is a phase transition line. For a strip geometry with the periodic boundary condition along the $x$-axis direction and the open boundary condition along the $y$-axis direction, if the sample width is sufficiently large (i.e., the lattice site numbers $n$ is much larger than superconducting coherence length $\xi_{0} \sim t /|\Delta|$ ), a pair of gapless chiral edge modes per boundary always present for $-4 t<\mu<0$ and $0<\mu<4 t$. In the former parameter region, the gapless points are present at $q=q_{x}=0$, and in the latter at $q= \pm \pi$. The
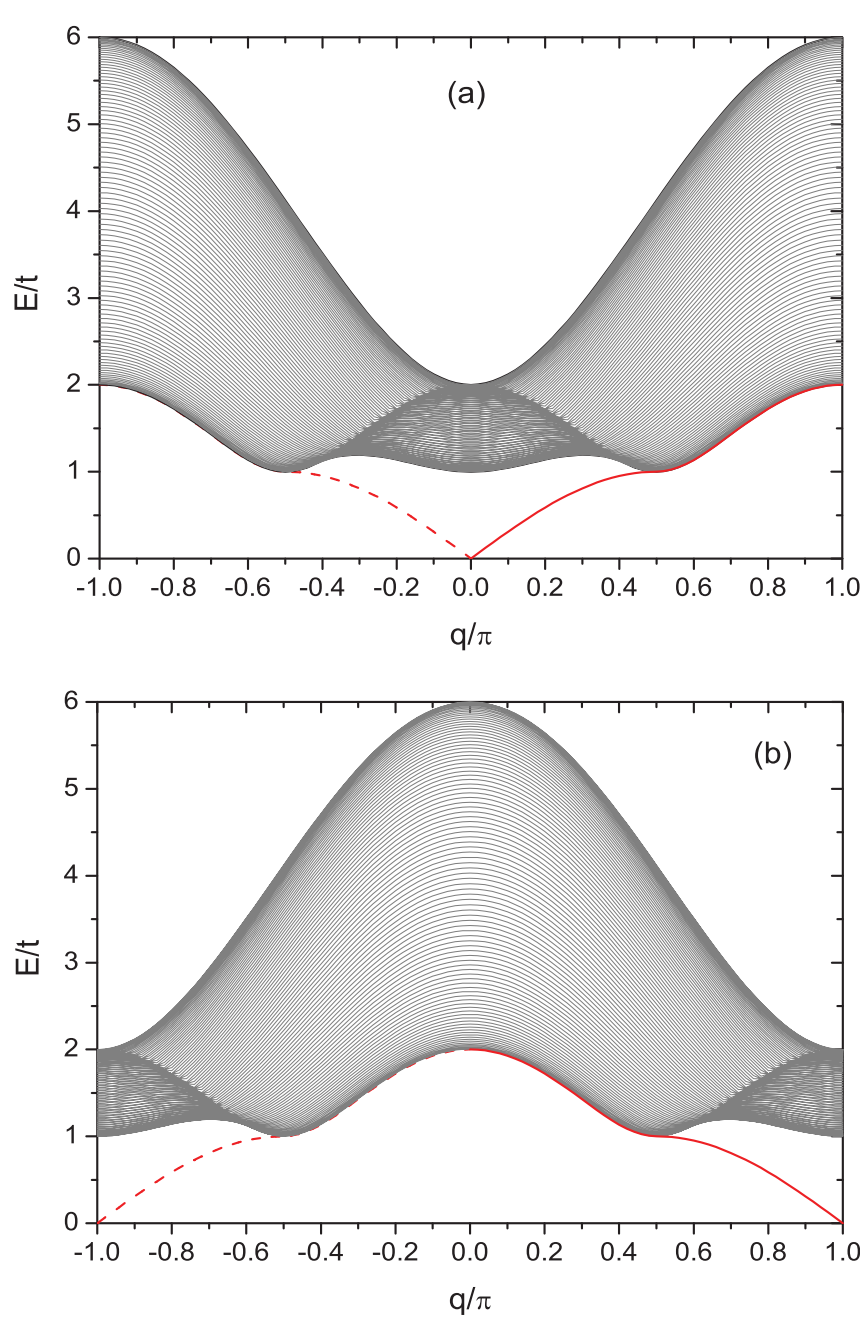

FIG. 1. (Color online) The excitation spectrum obtained by numerical diagonalization of the Hamiltonian Eq. (3) with the parameters $n=100, \Delta / t=0.5$. (a) $\mu / t=-2.0$; (b) $\mu / t=2.0$. The (red) solid and dashed lines correspond to the edge modes, which are localized at opposite boundaries of the sample, respectively.

excitation spectra obtained by numerical diagonalization of the Hamiltonian Eq. (3) with $n=100$ are plotted in Fig. 1, where the chiral edge modes are shown inside the bulk gap and the energies of chiral edge modes are $\pm 2|\Delta| \sin q$. It is interesting to note that different from the continuum model, the minimum of the edge dispersion shifts from $q=0$ to $q=\pi$ as one goes from negative to positive $\mu$ in the tight-binding model. This result can be understood based on the fact that if in the whole 1D Brillouin zone $(-\pi \leqslant q \leqslant \pi)$ the gapless points are present at both $q=0$ and $\pm \pi$, the edge modes with the spectrum $\pm 2|\Delta| \sin q$ would intersect Fermi energy (assumed inside the excitation gap) four times (an even multiple of two), which violates the topological stability. ${ }^{41-43}$ Hence, the edge modes must merge into the excitation spectrum at finite $q$, and the gapless points occur either at $q=0$ or $q=\pi$, but not at both. $^{44}$

As the sample width is decreased, due to the finite size effect, the wave functions of the edge modes on opposite edges overlap and mix, thus the spectrum reopens a gap. In Sec. III, we will focus on this issue. 


\section{MAJORANA EDGE STATES IN THE BOUNDARIES OF $p$-WAVE SUPERCONDUCTORS}

Here, we introduce a two-component field operator for each site $\alpha$ along the $y$-axis direction:

$$
\hat{d}_{\alpha}(q)=\frac{1}{2}\left[\begin{array}{l}
\gamma_{2 \alpha-1}(q) \\
\gamma_{2 \alpha}(q)
\end{array}\right] .
$$

The Hamiltonian Eq. (6) can be expressed in the terms of two component creation and annihilation operators $\hat{d}_{\alpha}^{\dagger}(q)$ and $\hat{d}_{\alpha}(q)$ as

$$
H=\sum_{q, \alpha}\left\{\hat{d}_{\alpha}^{\dagger}(q) \hat{\mathcal{E}} \hat{d}_{\alpha}(q)+\left[\hat{d}_{\alpha}^{\dagger}(q) \hat{\mathcal{T}} \hat{d}_{\alpha+1}(q)+\text { h.c. }\right]\right\},
$$

where

$$
\begin{gathered}
\hat{\mathcal{E}}=-2|\Delta| \sin q \sigma_{z}-(\mu+2 t \cos q) \sigma_{y}, \\
\hat{\mathcal{T}}=-t \sigma_{y}-i|\Delta| \sigma_{x},
\end{gathered}
$$

with $\sigma_{i}(i=x, y, z)$ being the Pauli matrices. We define the single particle states of the form

$$
\Psi(q)=\sum_{\alpha} \hat{d}_{\alpha}^{\dagger}(q) \psi_{\alpha}|0\rangle,
$$

where $|0\rangle$ is the vacuum state annihilated by the operator $\hat{d}_{\alpha}(q)$, and $\psi_{\alpha}=\left(\psi_{\alpha 1}, \psi_{\alpha 2}\right)^{T}$ is the two-component amplitude with index $\alpha$. An open-boundary condition $\psi_{\alpha=0}=\psi_{\alpha=n+1}=0$ is introduced along the sample width direction. The corresponding Schrödinger equation is given by

$$
\hat{\mathcal{E}}(q) \psi_{\alpha}+\hat{\mathcal{T}}(q) \psi_{\alpha+1}+\hat{\mathcal{T}}^{\dagger}(q) \psi_{\alpha-1}=\varepsilon(q) \psi_{\alpha} .
$$

Note that Eq. (17) is analog to the equation given in studying edge modes of topological insulators based on the tightbinding model. ${ }^{44-46}$

We study solutions of the form $\psi_{\alpha} \sim \lambda^{\alpha} \psi$ with $\lambda$ being a general complex number. ${ }^{47}$ First, substituting the ansatz into Eq. (17), we have

$$
\left[\hat{\mathcal{E}}(q)+\lambda \hat{\mathcal{T}}(q)+\lambda^{-1} \hat{\mathcal{T}}^{\dagger}(q)\right] \psi=\varepsilon(q) \psi .
$$

Thus, the secular equation gives an equation about $\lambda$ and $\varepsilon(q)$ :

$$
\begin{aligned}
& \left(t^{2}-|\Delta|^{2}\right) z^{2}+2 t(\mu+2 t \cos q) z \\
& \quad=\varepsilon^{2}-4|\Delta|^{2}\left(1+\sin ^{2} q\right)-(\mu+2 t \cos q)^{2},
\end{aligned}
$$

where $z=\left(\lambda+\lambda^{-1}\right)$. Note that if $\lambda$ is a solution, so is $\lambda^{-1}$. Thus, for every exponentially increasing solution, there exists another one which exponentially decreases. ${ }^{47}$ Four roots of $\lambda$ are given by

$$
\lambda_{1,2}=\frac{z_{ \pm}+\sqrt{z_{ \pm}^{2}-4}}{2}, \quad \lambda_{3,4}=\frac{z_{ \pm}-\sqrt{z_{ \pm}^{2}-4}}{2},
$$

where $z_{ \pm}$are two roots of $z$ in Eq. (19). We define two of four roots of $\lambda$ as $\lambda_{ \pm}$, which satisfy the relation $\left|\lambda_{ \pm}^{-1}\right|<1$, and then the other two roots of $\lambda$ are $\lambda_{ \pm}^{-1}$ satisfying $\left|\lambda_{ \pm}^{-1}\right|>1$.

Now we consider the edge states. For convenience, a symmetric boundary condition is used in the following, that is $\psi_{\tilde{\alpha}=-Y / 2}=\psi_{\tilde{\alpha}=Y / 2}=0$. Here, $Y=n+1$, and the new index $\tilde{\alpha}=\alpha-(n+1) / 2$. Thus, for the wave function $\psi_{\tilde{\alpha}}=$ $\left(\psi_{\tilde{\alpha} 1}, \psi_{\tilde{\alpha} 2}\right)^{T}$, we have an analytical expression: ${ }^{48}$

$$
\begin{aligned}
& \psi_{\tilde{\alpha} 1}=\tilde{c}_{+} f_{+}(q, \tilde{\alpha})+\tilde{c}_{-} f_{-}(q, \tilde{\alpha}), \\
& \psi_{\tilde{\alpha} 2}=\tilde{d}_{+} f_{+}(q, \tilde{\alpha})+\tilde{d}_{-} f_{-}(q, \tilde{\alpha}),
\end{aligned}
$$

where

$$
\begin{aligned}
& f_{+}(q, \tilde{\alpha})=\frac{\cosh \left(\tilde{\lambda}_{+} \tilde{\alpha}\right)}{\cosh \left(\tilde{\lambda}_{+} Y / 2\right)}-\frac{\cosh \left(\tilde{\lambda}_{-} \tilde{\alpha}\right)}{\cosh \left(\tilde{\lambda}_{-} Y / 2\right)}, \\
& f_{-}(q, \tilde{\alpha})=\frac{\sinh \left(\tilde{\lambda}_{+} \tilde{\alpha}\right)}{\sinh \left(\tilde{\lambda}_{+} Y / 2\right)}-\frac{\sinh \left(\tilde{\lambda}_{-} \tilde{\alpha}\right)}{\sinh \left(\tilde{\lambda}_{-} Y / 2\right)},
\end{aligned}
$$

with $\tilde{\lambda}_{ \pm}=\ln \lambda_{ \pm}$. The nontrivial solution for the coefficients $\tilde{c}_{ \pm}$and $\tilde{d}_{ \pm}$in the wave functions leads to a secular equation:

$$
\begin{aligned}
& \left(t^{2}-|\Delta|^{2}\right)\left(\cosh ^{2} \tilde{\lambda}_{+}+\cosh ^{2} \tilde{\lambda}_{-}\right)+2|\Delta|^{2} \\
& \quad=2 t^{2} \cosh \tilde{\lambda}_{+} \cosh \tilde{\lambda}_{-}-T|\Delta|^{2} \sinh \tilde{\lambda}_{+} \sinh \tilde{\lambda}_{-},
\end{aligned}
$$

with

$$
T=\frac{\tanh \left(\tilde{\lambda}_{+} Y / 2\right)}{\tanh \left(\tilde{\lambda}_{-} Y / 2\right)}+\frac{\tanh \left(\tilde{\lambda}_{-} Y / 2\right)}{\tanh \left(\tilde{\lambda}_{+} Y / 2\right)} .
$$

Equations (20) and (25) give the energy dispersions and the values of two characteristic quantities $\lambda_{ \pm}=e^{\tilde{\lambda}_{ \pm}}$.

When the sample width is sufficiently large, i.e., in the limit of $n \rightarrow \infty$, we can find two chiral edge modes with energy

$$
\varepsilon_{ \pm}(q)= \pm 2|\Delta| \sin q,
$$

and

$\lambda_{ \pm}=\frac{-(\mu+2 t \cos q) \pm \sqrt{(\mu+2 t \cos q)^{2}-4 t^{2}+4|\Delta|^{2}}}{2(t+|\Delta|)}$.

For nonzero $q$, the eigenstates for $\varepsilon_{ \pm}>0$ become concentrated on one edge or the other, depending on the sign of $q$, that is the left-moving and right-moving edge modes are localized at the opposite boundaries, respectively. ${ }^{2}$ Equation (27) is consistent with the result by numerical diagonalization (see Fig. 1). Interesting to note the cases of $q=0$ (for $\mu<0$ ) and $\pi$ (for $\mu>0$ ); one has zero-energy mode $\varepsilon(q=0 / \pi)=0$, which corresponds to the Majorana edge state in $p$-wave superconductors. ${ }^{2}$

Actually, there are no exact $\varepsilon=0$ modes for a finite-width sample. One of the key features for the solution of a finite width is the gap $E_{g}$ opening for the energy dispersion of the edge state. From Eqs. (20) and (25), one has

$$
\begin{aligned}
\varepsilon^{2}-4|\Delta|^{2} \sin ^{2} q= & 2 t(\mu+2 t \cos q)\left(\cosh \lambda_{+}+\cosh \lambda_{-}\right) \\
& +(\mu+2 t \cos q)^{2}+4 t^{2} \cosh \lambda_{+} \cosh \lambda_{-} \\
& -2 T|\Delta|^{2} \sinh \lambda_{+} \sinh \lambda_{-} ;
\end{aligned}
$$

thus, it can be found that a finite-energy gap $E_{g}$ at $q=0$ (for the case of $\mu<0$ ) is approximately

$$
E_{g} \simeq|\Delta| \sqrt{\left|\frac{2 \mu(\mu+4 t)}{t^{2}-|\Delta|^{2}}\right|} e^{-n / l_{0}},
$$


and at $q=\pi$ (for the case of $\mu>0$ )

$$
E_{g} \simeq|\Delta| \sqrt{\left|\frac{2 \mu(\mu-4 t)}{t^{2}-|\Delta|^{2}}\right|} e^{-n / l_{0}},
$$

where $l_{0}^{-1}=\min \left(|\ln | \lambda_{+}||,|\ln | \lambda_{-}||\right)$, and $l_{0}$ denotes the localization length of the edge mode. The gap $E_{g}$ will decrease exponentially with increase of the width of the strip.

\section{MAJORANA END STATES IN QUASI-1D $p$-WAVE SUPERCONDUCTORS}

In a recent work by Potter and Lee ${ }^{34}$ two well-isolated Majorana end states localized at opposite ends of the strip geometry have been obtained by numerical diagonalization of the Hamiltonian Eq. (1). Here we will give the phase diagrams of the presence of Majorana end modes in quasi-1D $p$-wave superconductors by using topological arguments due to Kitaev. ${ }^{5}$ To this aim, we consider the $2 n \times 2 n$ matrix $B(q)$ in the Hamiltonian Eq. (6). The matrix $B$ is an antisymmetric matrix when $q$ is equal to zero or $\pi$, such that we can calculate the Pfaffians $\operatorname{Pf} B(0)$ and $\operatorname{Pf} B(\pi)$. The topological property of the system described by the Hamiltonian Eq. (6) is characterized by a $Z_{2}$ topological index (Majorana number) $\mathcal{M}$ :

$$
\mathcal{M}=\operatorname{sgn}[\operatorname{Pf} B(0)] \operatorname{sgn}[\operatorname{Pf} B(\pi)]= \pm 1,
$$

where +1 corresponds to topologically trivial states and -1 to topologically nontrivial states (i.e., the existence of zero mode Majorana end states). ${ }^{5,28,32,36}$

For the simplest case, there is only one lattice site along the $y$-axis direction (i.e., $n=1$ ). This case is just the Kitaev original model. ${ }^{5}$ Two $2 \times 2$ antisymmetric matrices are

$$
B_{n=1}(0 / \pi)=\left[\begin{array}{lc}
0 & \mu \pm 2 t \\
-(\mu \pm 2 t) & 0
\end{array}\right],
$$

and $\operatorname{Pf} B_{n=1}(0 / \pi)=\mu \pm 2 t$, where "+" and "-" correspond to the cases of $q=0$ and $\pi$, respectively. The Majorana number for the case of the strict 1D limit is

$$
\mathcal{M}_{n=1}=\operatorname{sgn}(\mu+2 t) \operatorname{sgn}(\mu-2 t) ;
$$

thus, we have the topologically nontrivial condition

$$
2|t|>|\mu|(|\Delta| \neq 0) .
$$

Equation (35) is the result of Kitaev, ${ }^{5}$ who demonstrated for a long open chain (in the limit of $L \rightarrow \infty$ ) there are zero-energy Majorana end states localized near per boundary point under the condition Eq. (35). However, if the chain length $L$ is finite, there is a weak interaction between two unpaired Majorana fermions. ${ }^{5}$ Following the method given in Sec. III, we can also obtain the energy gap opened due to the finite-size effect approximately as

$$
\Delta_{g} \simeq|\Delta| \sqrt{\left|\frac{2\left(4 t^{2}-\mu^{2}\right)}{t^{2}-|\Delta|^{2}}\right|} e^{-L / \tilde{l}_{0}},
$$
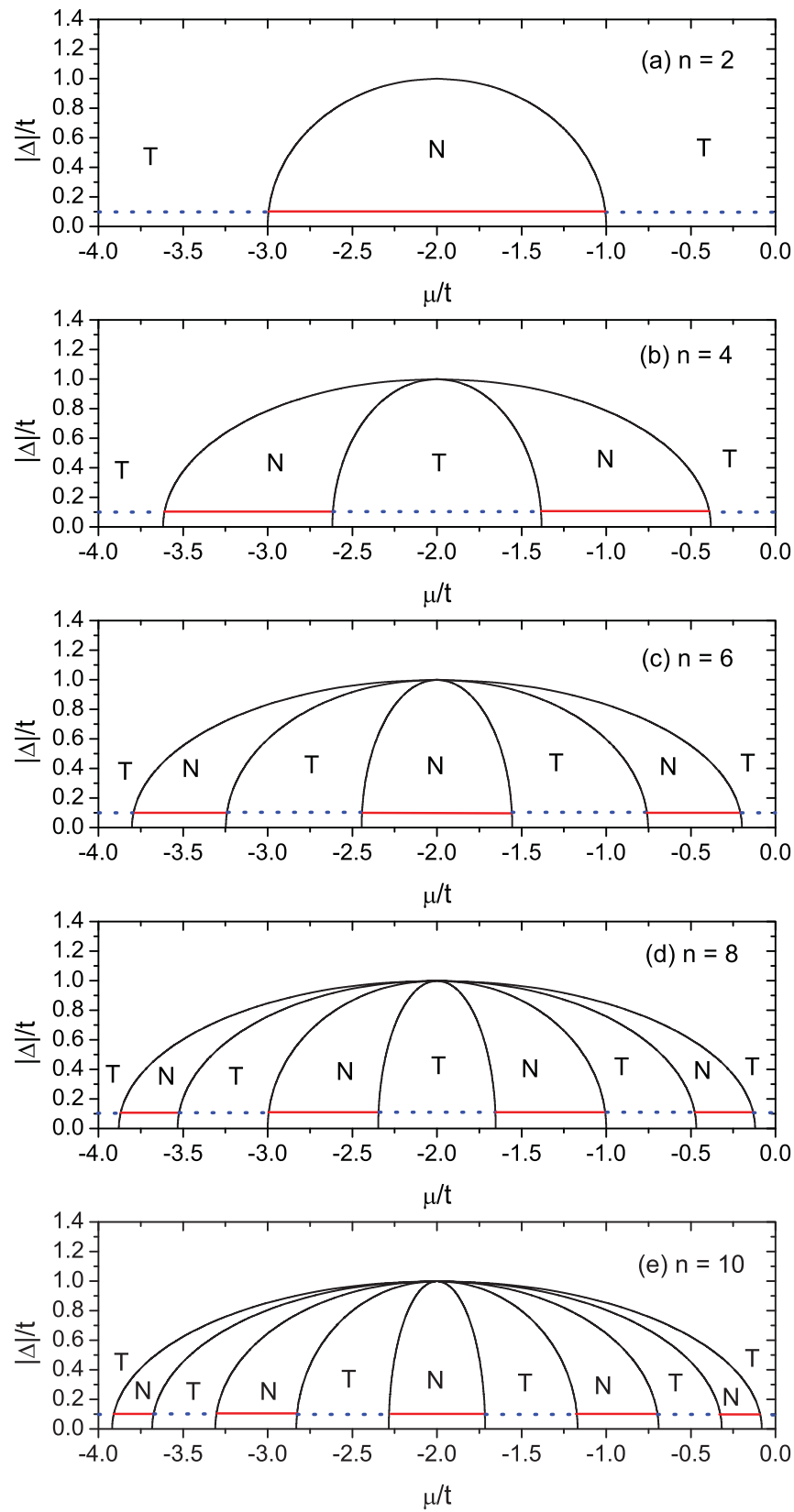

FIG. 2. (Color online) Phase diagram for the quasi-1D $p$-wave superconductor model as a function of the $p$-wave pairing amplitude and chemical potential for even lattice site numbers $n$ along the $y$-axis direction. "N" denotes the topologically nontrivial region in the presence of zero-mode Majorana end states, and " $\mathrm{T}$ " denotes the topologically trivial region without zero-mode states. When $|\Delta| / t=$ 0.1 , the solid (red) lines and dotted (blue) lines guide the values of $\mu / t$, corresponding to the topologically nontrivial and trivial phases, respectively. (a) $n=2$; (b) $n=4$; (c) $n=6$; (d) $n=8$; (e) $n=10$.

where $\tilde{l}_{0}^{-1}=\min \left(|\ln | x_{+}||,|\ln | x_{-}||\right)$, with

$$
x_{ \pm}=\frac{-\mu \pm \sqrt{\mu^{2}-4 t^{2}+4|\Delta|^{2}}}{2(t+|\Delta|)} .
$$

Here, $\tilde{l}_{0}$ indicates the localization length of the Majorana end states. Thus, the energy gap $\Delta_{g}$ vanishes as $\exp \left(-L / \tilde{l}_{0}\right)$ in an open chain. ${ }^{5}$ 

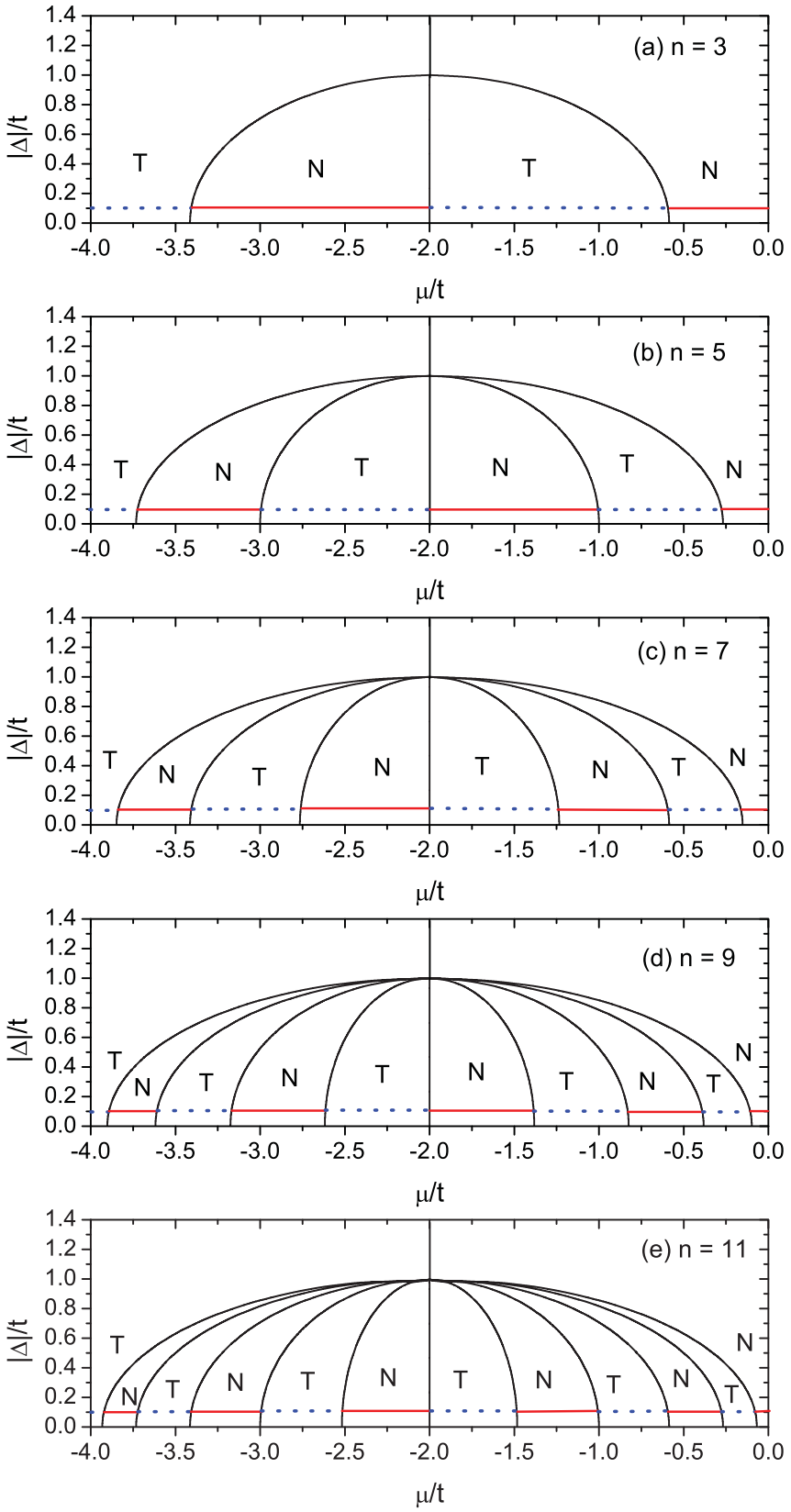

FIG. 3. (Color online) Phase diagram for the quasi-1D $p$-wave superconductor model as a function of the $p$-wave pairing amplitude and chemical potential for odd lattice site numbers $n$ along the $y$ axis direction. "N" denotes the topologically nontrivial region in the presence of zero-mode Majorana end states, and " $\mathrm{T}$ " denotes the topologically trivial region without zero-mode states. When $|\Delta| / t=$ 0.1 , the solid (red) lines and dotted (blue) lines guide the values of $\mu / t$, corresponding to the topologically nontrivial and trivial phases, respectively. (a) $n=3$; (b) $n=5$; (c) $n=7$; (d) $n=9$; (e) $n=11$.

For the case of $n=2$, the lattice site numbers along the $y$-axis direction are two. Two $4 \times 4$ antisymmetric matrices are

$$
\begin{aligned}
B_{n=2}(0 / \pi) & \\
= & {\left[\begin{array}{lccc}
0 & \mu \pm 2 t & 0 & t-|\Delta| \\
-(\mu \pm 2 t) & 0 & -(t+|\Delta|) & 0 \\
0 & t+|\Delta| & 0 & \mu \pm 2 t \\
-(t-|\Delta|) & 0 & -(\mu \pm 2 t) & 0
\end{array}\right] . }
\end{aligned}
$$

The direct calculation yields the Pfaffians $\operatorname{Pf} B_{n=2}(0 / \pi)$ :

$$
\operatorname{Pf} B_{n=2}(0 / \pi)=(\mu \pm 2 t)^{2}+|\Delta|^{2}-t^{2} .
$$

For the larger lattice site numbers $n(\geqslant 3), \operatorname{Pf} B_{n}(0 / \pi)$ can be also calculated analytically, and we obtain a recursion relation

$$
\operatorname{Pf} B_{n}(0 / \pi)=a_{ \pm} \operatorname{Pf} B_{n-1}(0 / \pi)+b \operatorname{Pf} B_{n-2}(0 / \pi),
$$

where $a_{ \pm}=\mu \pm 2 t$ and $b=|\Delta|^{2}-t^{2}$. We further solve Eq. (40) and give an analytic formula for $\operatorname{Pf} B_{n}(0 / \pi)$ :

$$
\operatorname{Pf} B_{n}(0 / \pi)=\frac{\left(r_{1}^{n+1}-r_{2}^{n+1}\right)}{\sqrt{a_{ \pm}^{2}+4 b}},
$$

where

$$
r_{1}=\frac{a_{ \pm}+\sqrt{a_{ \pm}^{2}+4 b}}{2}, \quad r_{2}=\frac{a_{ \pm}-\sqrt{a_{ \pm}^{2}+4 b}}{2} .
$$

According to the Pfaffians $\operatorname{Pf} B_{n}(0 / \pi)$, one can compute $\mathcal{M}$ as a function of the physical parameters and then plot the phase diagram showing a sequence of topological phase transition for different lattice site numbers $n$. Figures 2 and 3 plot the phase diagrams for the even and odd lattice site numbers $n$ along the $y$-axis direction, respectively. The phase diagrams of this tight-binding model have the symmetry on positive and negative $\mu$ values; thus, here we only plot on negative $\mu$ values because the other part on positive $\mu$ values is a mirror image. However, this $\mu \rightarrow-\mu$ symmetry is not generic to models with, say, next-nearest-neighbor hopping or nextnearest-neighbor pairing.

We now analyze these phase diagrams. First, it is observed that for the given value of $|\Delta| / t$, the topologically nontrivial and trivial phases alternate with the variation of the value of $\mu / t$. As an example, the case of $|\Delta| / t=0.1$ is shown in Figs. 2 and 3. From Figs. 2 and 3, it is shown that the

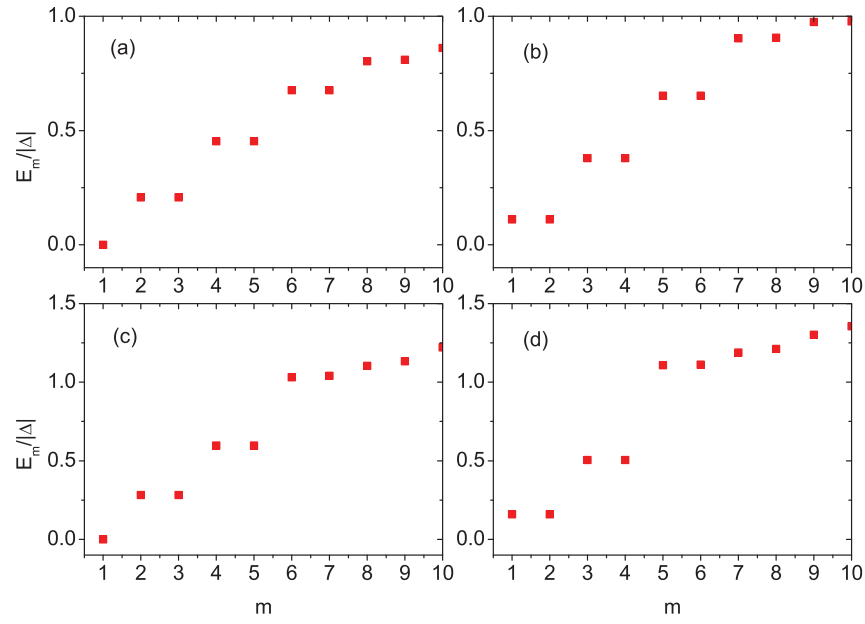

FIG. 4. (Color online) Excitation spectrum $E_{m} /|\Delta|$ for a squarelattice Kitaev model obtained by numerical diagonalization of the Hamiltonian Eq. (1) with parameters: $L=100, n=10,|\Delta| / t=0.1$. $m$ labels eigenvalues of the Hamiltonian Eq. (1). Here, only the partial low excitation energies are presented. (a) $\mu / t=-1.0\left(E_{1} /|\Delta|=\right.$ $\left.1.09 \times 10^{-5}\right)$; (b) $\mu / t=-1.5\left(E_{1} /|\Delta|=0.1116\right)$; (c) $\mu / t=-2.0$ $\left(E_{1} /|\Delta|=1.54 \times 10^{-5}\right)$; (e) $\mu / t=-2.5\left(E_{1} /|\Delta|=0.1595\right)$. 


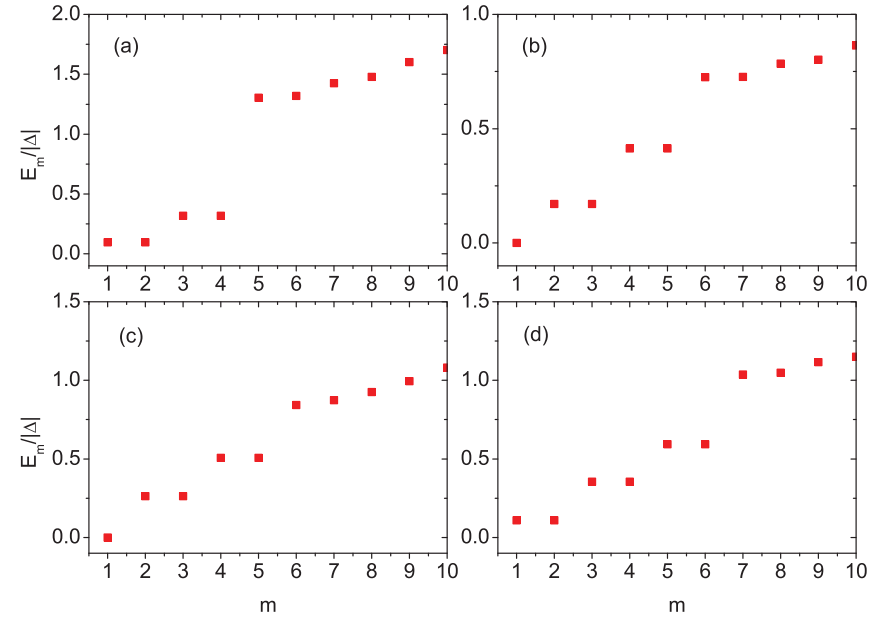

FIG. 5. (Color online) Excitation spectrum $E_{m} /|\Delta|$ for a squarelattice Kitaev model obtained by numerical diagonalization of the Hamiltonian Eq. (1) with parameters: $L=100,|\Delta| / t=0.1, \mu / t=$ -1.1. $m$ labels eigenvalues of the Hamiltonian Eq. (1). Here, only the partial low-excitation energies are presented. (a) $n=6\left(E_{1} /|\Delta|=\right.$ $0.0966)$; (b) $n=7\left(E_{1} /|\Delta|=8.21 \times 10^{-6}\right)$; (c) $n=8\left(E_{1} /|\Delta|=\right.$ $\left.7.39 \times 10^{-6}\right)$; (e) $n=9\left(E_{1} /|\Delta|=0.1102\right)$.

phase diagrams have different properties for the even and odd lattice site numbers $n$. Such an odd-even effect should be an artifact of the tight-binding model and may not be generic to all quasi-1D $p$-wave supercondurtors, since the order parameter for Cooper pairing is presented as a model parameter, not given by a self-consistent mean field calculation. Even in a realistic material, the oscillation of the critical temperature with the monolayer number of the sample was observed experimentally as quantum confinement effect. ${ }^{49}$ It is not clear what the real meaning of odd and even $n$ in this model calculation, which may have no counterpart in the continuous model as a long wave length limit. However, the oscillation of the presence and absence of the zero mode still clearly exhibits in the continuous model as quantum confinement effect.

According to the topological argument above, the topologically nontrivial phase corresponds to the existence of the zero energy mode, which can be justified by the excitation spectrum obtained by the numerical diagonalization of the Hamiltonian Eq. (1). According to the work by Potter and Lee, ${ }^{34}$ for sufficiently long samples $\left(L \gg e^{n / \xi_{0}}\right)$, one can find spatially isolated zero-energy Majorana end states localized at opposite ends of the sample with the strip geometry. In Fig. 2(e) (with $n=10$ ), we see that if $|\Delta| / t$ is fixed at 0.1 , the cases of $\mu / t=-1.0$ and -2.0 correspond to the topologically nontrivial phase; the cases of $\mu / t=-1.5$ and -2.5 to the topologically trivial phase. For the above four parameter cases, the excitation spectra of the Hamiltonian Eq. (1) with $L=100$ and $n=10$ are plotted in Fig. 4. Numerical diagonalizations show that the zero-energy modes indeed exist in the cases of $\mu / t=-1.0$ and -2.0 ; there are no zero-energy modes in the cases of $\mu / t=-1.5$ and -2.5 . The conclusions are consistent with the results by Potter and Lee. ${ }^{34}$

We note also an interesting property from the phase diagrams. For some given parameters, the topological property of a strip geometry will change in an oscillatory way with respect to the sample width. For instance, in Fig. 2, when the parameters $|\Delta| / t=0.1$ and $\mu / t=-2.0$ are fixed, the topologically nontrivial and trivial phases alternate when the lattice site numbers $n$ (even numbers) along the $y$-axis direction change from $n=2$ to $n=10$. While in the cases of the odd $n, \mu / t=-2.0$ is a topological phase transition line (see Fig. 3). As another example, we take the parameters $|\Delta| / t=0.1, \mu / t=-1.1$, and then from Figs. 2 and 3 it is presented that the topologically nontrivial phases exist in the cases of $n=2,4,5,7,8,10$, and 11; while the cases of $n=3,6$, and 9 correspond to the topologically trivial phases. In Fig. 5, the excitation spectra for four different sample widths ( $n=6,7,8$, and 9) are shown. For the cases $n=7$ and 8, there are zero-energy modes in the given parameters; while the zero-energy modes disappear in the cases of $n=6$ and 9 . Thus, the topological property obtained by the phase diagrams is consistent with the results by numerical diagonalization.

\section{CONCLUSIONS}

In this paper, we investigate crossover from Majorana edge to end states in an ideal spinless quasi-1D $p$-wave superconductor based on the tight-banding model. We found the existence of Majorana edge modes when the sample width is sufficiently large, and then through analytical solutions in a model calculation, two Majorana fermions at the two edges can couple together to produce a gap in the excitation spectrum for a strip of finite width under the periodic boundary condition along the longitudinal direction. We calculate $Z_{2}$ topological index in the quasi-1D sample and plot the phase diagrams of the presence of Majorana end modes. By analyzing the phase diagrams, we find that for some given parameters the topological property of a strip geometry will change in an oscillatory way with respect of the sample width.

\section{ACKNOWLEDGMENTS}

This work was supported by the Research Grant Council of Hong Kong under Grant Nos. HKU7051/10P and HKUST3/CRF/09. Z.B. was supported by the National Natural Science Foundation of China (Grant No. 10974046), Hubei Provincial Natural Science Foundation of China (Grant No. 2009CDB360), and the Key Project of Education Department of Hubei Province of China (Grant No. D20101004).
${ }^{1}$ F. Wilczek, Nature Phys. 5, 614 (2009).

${ }^{2}$ N. Read and D. Green, Phys. Rev. B 61, 10267 (2000).

${ }^{3}$ D. A. Ivanov, Phys. Rev. Lett. 86, 268 (2001).

${ }^{4}$ C. Nayak, S. H. Simon, A. Stern, M. Freedman, and S. Das Sarma, Rev. Mod. Phys. 80, 1083 (2008).
${ }^{5}$ A. Y. Kitaev, Phys. Usp. 44, 131 (2001).

${ }^{6}$ A. Stern, F. von Oppen, and E. Mariani, Phys. Rev. B 70, 205338 (2004).

${ }^{7}$ A. R. Akhmerov, Phys. Rev. B 82, 020509(R) (2010). 
${ }^{8}$ F. Hassler, A. R. Akhmerov, C.-Y. Hou, and C. W. J. Beenakker, New J. Phys. 12, 125002 (2010).

${ }^{9}$ G. Moore and N. Read, Nucl. Phys. B 360, 362 (1991).

${ }^{10}$ M. Greiter, X. G. Wen, and F. Wilczek, Nucl. Phys. B 374, 567 (1992).

${ }^{11}$ S. Das Sarma, C. Nayak, and S. Tewari, Phys. Rev. B 73, 220502(R) (2006).

${ }^{12}$ R. Roy, Phys. Rev. Lett. 105, 186401 (2010).

${ }^{13}$ G. E. Volovik, JETP Lett. 70, 609 (1999).

${ }^{14} \mathrm{G}$. E. Volovik, The Universe in a Helium Droplet (Oxford University Press, Oxford, 2003).

${ }^{15}$ M. A. Silaev and G. E. Volovik, J. Low Temp. Phys. 161, 460 (2010).

${ }^{16}$ L. Fu and C. L. Kane, Phys. Rev. Lett. 100, 096407 (2008).

${ }^{17}$ L. Fu and C. L. Kane, Phys. Rev. B 79, 161408(R) (2009).

${ }^{18}$ A. R. Akhmerov, J. Nilsson, and C. W. J. Beenakker, Phys. Rev. Lett. 102, 216404 (2009).

${ }^{19}$ Y. Tanaka, T. Yokoyama, and N. Nagaosa, Phys. Rev. Lett. 103, 107002 (2009).

${ }^{20}$ K. T. Law, P. A. Lee, and T. K. Ng, Phys. Rev. Lett. 103, 237001 (2009).

${ }^{21}$ J. Linder, Y. Tanaka, T. Yokoyama, A. Sudbø, and N. Nagaosa, Phys. Rev. Lett. 104, 067001 (2010).

${ }^{22}$ V. Shivamoggi, G. Refael, and J. E. Moore, Phys. Rev. B 82, 041405(R) (2010).

${ }^{23}$ P. A. Ioselevich and M. V. Feigel'man, Phys. Rev. Lett. 106, 077003 (2011).

${ }^{24}$ J. Nilsson, A. R. Akhmerov, and C. W. J. Beenakker, Phys. Rev. Lett. 101, 120403 (2008).

${ }^{25}$ J. D. Sau, R. M. Lutchyn, S. Tewari, and S. Das Sarma, Phys. Rev. Lett. 104, 040502 (2010).

${ }^{26}$ J. Linder and A. Sudbø, Phys. Rev. B 82, 085314 (2010).

${ }^{27}$ J. Alicea, Phys. Rev. B 81, 125318 (2010).

${ }^{28}$ R. M. Lutchyn, J. D. Sau, and S. Das Sarma, Phys. Rev. Lett. 105, 077001 (2010).
${ }^{29}$ Y. Oreg, G. Refael, and F. von Oppen, Phys. Rev. Lett. 105, 177002 (2010).

${ }^{30}$ J. Alicea, Y. Oreg, G. Refael, F. von Oppen, and M. P. A. Fisher, Nature Phys. 7, 412 (2011).

${ }^{31}$ S. Fujimoto, Phys. Rev. B 77, 220501(R) (2008).

${ }^{32}$ P. Ghosh, J. D. Sau, S. Tewari, and S. Das Sarma, Phys. Rev. B 82, 184525 (2010)

${ }^{33}$ S.-L. Zhu, L.-B. Shao, Z. D. Wang, and L.-M. Duan, Phys. Rev. Lett. 106, 100404 (2011).

${ }^{34}$ A. C. Potter and P. A. Lee, Phys. Rev. Lett. 105, 227003 (2010).

${ }^{35}$ M. Wimmer, A. R. Akhmerov, M. V. Medvedyeva, J. Tworzydło, and C. W. J. Beenakker, Phys. Rev. Lett. 105, 046803 (2010).

${ }^{36}$ R. M. Lutchyn, T. D. Stanescu, and S. Das Sarma, Phys. Rev. Lett. 106, 127001 (2011).

${ }^{37}$ A. C. Potter and P. A. Lee, Phys. Rev. B 83, 094525 (2011).

${ }^{38}$ K. T. Law and P. A. Lee, e-print arXiv:1103.5013v1 (unpublished).

${ }^{39}$ R. M. Lutchyn and M. P. A. Fisher, e-print arXiv:1104.2358v1 (unpublished).

${ }^{40}$ Similar discussions in Secs. II and III were presented in Ref. 35, in which Appendix $\mathrm{C}$ of the supplement presents analytic expressions for the decaying edge modes in the continuum limit and also chemical potentials where topological transitions occur in the quai-1D systems have been given.

${ }^{41}$ C. Wu, B. A. Bernevig, and S. C. Zhang, Phys. Rev. Lett. 96, 106401 (2006).

${ }^{42}$ H. B. Nielsen and M. Ninomiya, Nucl. Phys. B 185, 20 (1981).

${ }^{43}$ H. B. Nielsen and M. Ninomiya, Nucl. Phys. B 193, 173 (1981).

${ }^{44}$ S. Mao, Y. Kuramoto, K. I. Imura, and A. Yamakage, J. Phys. Soc. Jpn. 79, 124709 (2010).

${ }^{45}$ S. Mao and Y. Kuramoto, Phys. Rev. B 83, 085114 (2011).

${ }^{46}$ M. König, H. Buhmann, L. W. Molenkamp, T. Hughes, C.-X. Liu, X.-L. Qi, and S.-C. Zhang, J. Phys. Soc. Jpn. 77, 031007 (2008).

${ }^{47}$ M. Creutz and I. Horváth, Phys. Rev. D 50, 2297 (1994).

${ }^{48}$ B. Zhou, H.-Z. Lu, R.-L. Chu, S.-Q. Shen, and Q. Niu, Phys. Rev. Lett. 101, 246807 (2008).

${ }^{49}$ Y. Guo et al., Science 306, 1915 (2004). 